\title{
Síndrome de compresión traqueal por arteria innominada: Casos clínico-radiológicos
}

\section{Dres. Stefhanie Nayar $E^{(1)}$, Karla Moënne $B^{(2)}$, Carolina Pérez $S^{(2)}$, Jorge Mackenney $P^{(3)}$.}

1 Becada Broncopulmonar Infantil, Universidad de Chile Campus Norte. Santiago, Chile.

2 Radióloga Infantil, Clínica Las Condes. Santiago, Chile.

3 Pediatra Broncopulmonar, Clínica Las Condes y Universidad de Chile. Santiago, Chile.

\section{Syndrome of tracheal compression by the innominate artery: clinical-radiological cases}

\begin{abstract}
Innominate artery compression of the trachea, which is included within the vascular rings, although rare can cause serious respiratory disorders in childhood even putting at risk the child's life. Most cases are asymptomatic but can also occur with cough, stridor, cyanosis and/or apneas. Diagnostic images play a fundamental role, specially computed tomography and magnetic resonance imaging that provide detailed anatomical information. Bronchoscopy allows confirmation of the diagnosis and assesses the severity of the disorder; moreover, this procedure is also used as support in surgery (aortopexy). Conservative treatment is indicated in less severe cases. Two cases of pediatric patients are presented, one a newborn with severe symptoms and an asymptomatic lactating infant.
\end{abstract}

Keywords: Airway, Children, Innominate artery, Tracheal compression.

Resumen: La compresión traqueal por arteria innominada, que se incluye dentro de los anillos vasculares, aunque es poco frecuente puede provocar trastornos respiratorios graves en la infancia poniendo incluso en riesgo la vida del niño. La mayoría de los casos son asintomáticos aunque también pueden presentarse con tos, estridor, cianosis y/o apneas. Las imágenes diagnósticas cumplen un rol fundamental, destacando la tomografía computarizada y la resonancia magnética que otorgan información anatómica detallada. La fibrobroncoscopía permite confirmar el diagnóstico y estimar la severidad de la alteración; por otra parte, este procedimiento también se utiliza como apoyo en la cirugía (aortopexia). El manejo conservador está indicado en los casos menos severos. Se presentan 2 casos de pacientes pediátricos, un recién nacido con síntomas severos y un lactante menor asintomático.

Palabras clave: Arteria innominada, Compresión traqueal, Niños, Vía aérea.

Nayar S, Moënne K, Pérez C, Mackenney J. Síndrome de compresión traqueal por arteria innominada: Casos clínicoradiológicos. Rev Chil Radiol 2015; 21(2): 54-57.

Correspondencia: Dra. Stefhanie NayarE. / stefhaniealex@gmail.com

Trabajo recibido el 04 de septiembre de 2014. Aceptado para publicación 06 de julio de 2015.

\section{Introducción}

Las anomalías vasculares congénitas del arco aórtico, incluyendo los anillos vasculares, representan alrededor del $1 \%$ de todas las anomalías cardiovasculares congénitas ${ }^{(1)}$ y pueden comprimir la tráquea, el esófago o ambos órganos ${ }^{(2,3)}$. Es una causa poco común de trastornos respiratorios en la infancia, que puede resultar en una enfermedad grave e incluso poner en riesgo la vida del niño(4).

La compresión traqueal por arteria innominada fue descrita por primera vez en el año 1948, por
Gross y Neuhauser ${ }^{(5)}$. Embriológicamente esta alteración se produce por una diferenciación anormal del tercer par de arcos aórticos, provocando que el arco innominado cruce por delante de la tráquea después de emerger del arco aórtico en un punto más distal que lo habitual, por lo que también se denomina como síndrome de arteria innominada aberrante (Figura 1) (6,9) $^{(6 i}$ Sien esta alteración se incluye dentro de los anillos vasculares, no corresponde a un anillo vascular verdadero, ya que la compresión de la tráquea es solamente por vía anterior. La inciden- 
cia de esta malformación congénita es controversial, reportándose hasta en un 3\% en la población general, en sujetos mayoritariamente asintomáticos ${ }^{(4-7)}$.

Cabe destacar que en 1998 la Nómina Anatómica, que es redactada por un grupo de anatomistas desde 1950 y actualiza la nomenclatura de términos anatómicos, denominó a la arteria innominada como tronco braquiocefálico aunque la gran mayoría de la literatura continúa refiriéndose a esta entidad con Síndrome de compresión por arteria innominada ${ }^{(10)}$.

Los niños con síndrome de arteria innominada aberrante pueden presentar otras anomalías congénitas asociadas, entre las que destaca la laringotraqueomalacia primaria, que con frecuencia está presente en los casos sintomáticos ${ }^{(3,4)}$.

En el año 2013, Wine et al., describieron la existencia de comorbilidades en una serie de 26 niños con compresión traqueal por arteria innominada que requirieron tratamiento quirúrgico, reportando: laringomalacia (19\%), prolapso de la epiglotis y base de la lengua (12\%), estenosis subglótica (23\%), hipertrofia adenoidea (4\%) y traqueobroncomalacia $(27 \%)^{(8)}$.

A continuación describiremos dos casos pediátricos de compresión traqueal por arteria innominada, con especial énfasis en los hallazgos imaginológicos.

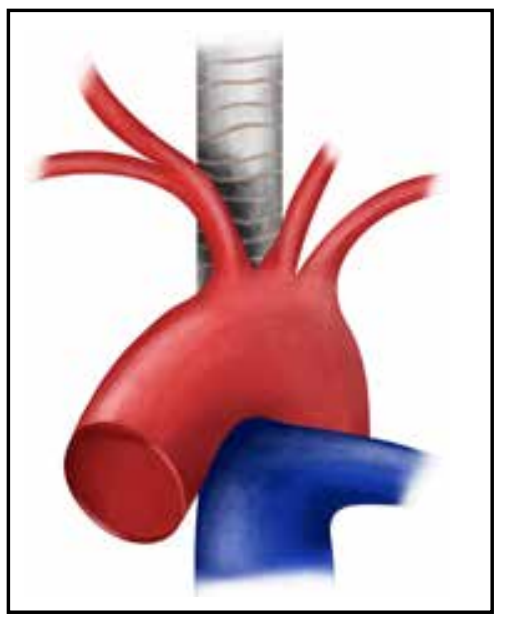

Figura 1. Esquema anatómico de compresión traqueal por arteria innominada.

\section{Caso $\mathrm{N}^{\circ} 1$}

Recién nacido de término, 40 semanas adecuado para la edad gestacional, sexo femenino, producto de parto normal, sin antecedentes patológicos prenatales. El segundo día de vida se observó aparición de estridor bimodal (inspiratorio y espiratorio), de tonalidad grave, que desaparece al dormir y aumenta con el llanto, asociándose a cianosis facial y disnea severa. Se efectuó radiografía de tórax AP portátil que no demostró alteraciones.

Al tercer día de vida fue evaluado por otorrinolaringólogo impresionando parálisis de cuerda vocal izquierda. Se solicitó ecocardiografía y tomografía computarizada (TC) de cuello y tórax. La ecocardiografía y la TC de cuello resultaron normales. En TC de tórax, efectuada con contraste endovenoso y sedación con máscara laríngea, se demostró presencia de compresión ánterolateral derecha de la tráquea, en relación a arteria innominada (Figura 2).

Se realizó fibrobroncoscopía que evidenció discreta menor movilidad de la cuerda vocal izquierda, que desapareció al finalizar el procedimiento y superficializar la sedación, observando abducción normal de ambas cuerdas vocales; la tráquea alta apareció normal y se observó compresión pulsátil de la cara anterior en la unión del tercio superior y el tercio medio de la tráquea, con disminución del lumen estimada como cercana al $50 \%$, que aumentaba a colapso casi completo al someter al paciente a presión negativa intratraqueal (Figura 3).

Si bien se observó regresión parcial espontánea de la sintomatología, se analizó el caso en reunión multidisciplinaria y se decidió que la clínica del paciente ameritaba tratamiento quirúrgico por tratarse de un estridor sintomático severo con evidencias de importante compresión traqueal asociada a malacia localizada. La paciente fue sometida a aortopexia sin incidentes a los 17 días de vida, comprobando bajo visión directa de fibrobroncoscopía la recuperación del lumen traqueal, sin evidencias de colapso; fue dada de alta en buenas condiciones a los 21 días de vida.
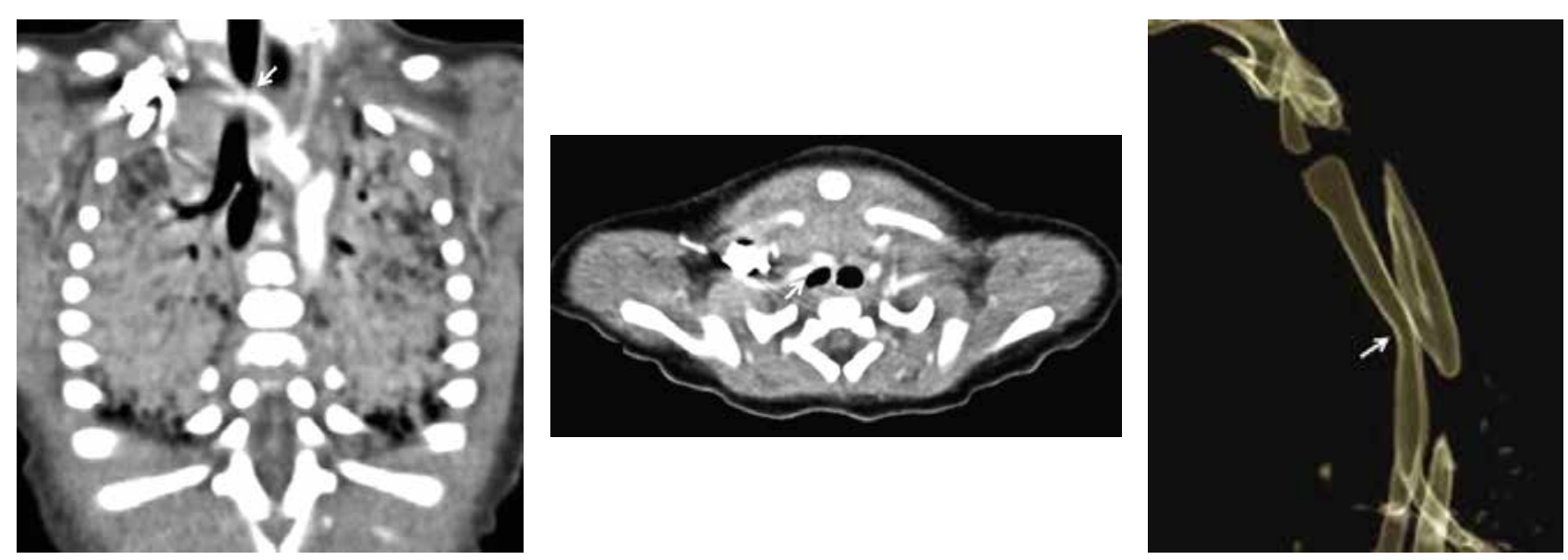

Figura 2. Imágenes de Caso $N^{\circ}$. TC de tórax con contraste evidencia compresión e incurvación de tráquea por arteria innominada (flechas); a) Reconstrucción coronal, b) Corte axial, c) Reconstrucción volumétrica sagital. 


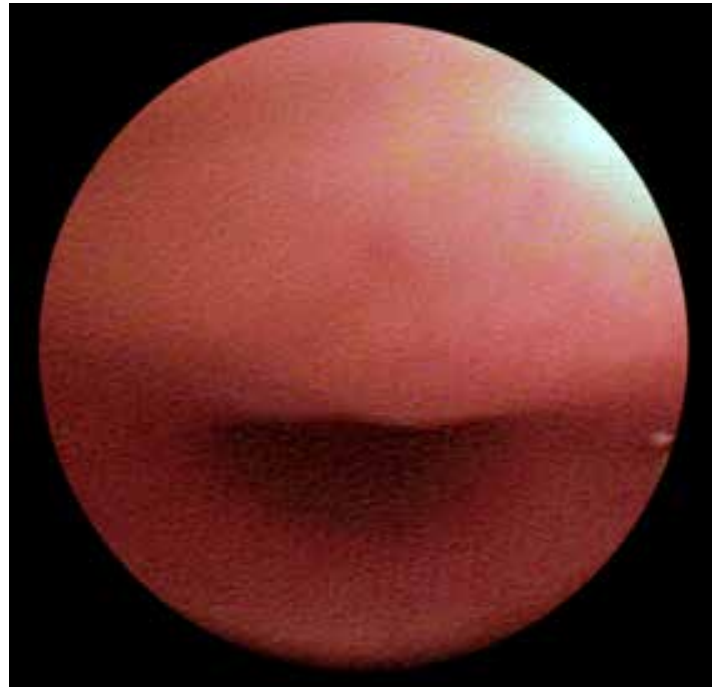

Figura 3. Imagen de fibrobroncoscopía evidencia compresión extrínseca anterior del lumen traqueal.

\section{Caso $\mathrm{N}^{\circ} 2$}

Antecedente de recién nacido de término, sin patología perinatal. En la evaluación de desarrollo psicomotor efectuada en control habitual se pesquisó hipertonía global y tendencia a posicionar cabeza hacia la derecha, sin otros síntomas asociados. Fue derivado a neurología infantil a los 3 meses de vida donde se solicitó TC de cerebro y columna cervical, para estudiar posibles causas de hipertonía y tortícolis.

En la TC de cuello se describió que el tronco arterial braquiocefálico o arteria innominada cursaba de izquierda a derecha provocando un desplazamiento posterior y angulación traqueal, sin disminución significativa de su lumen (Figura 4). Por tratarse de un paciente asintomático respiratorio, se estimó la alteración como un hallazgo que no requería tratamiento alguno.

\section{Discusión}

La presentación clínica de la compresión traqueal por arteria innominada es variada; si bien predominan los sujetos asintomáticos, también puede presentarse con diversos síntomas respiratorios entre los que destacan: tos, estridor, cianosis y/o apneas, ameritando en estos casos un estudio completo de la vía aérea. Gardella et al., (2010) estudiaron un grupo de 28 pacientes con esta patología, cuyas edades fluctuaban entre un mes y trece años, describiendo como síntomas más frecuentes: apneas reflejas, infecciones respiratorias bajas recurrentes, tos crónica intratable, disnea, estridor y sibilancias. Los autores no observaron diferencia de los síntomas según edad, excepto en apneas y episodios de ALTE (Apparent Life Threatening Event), que fueron más frecuentes en los menores de dos años y en presencia de tos crónica, que se observó en niños mayores. El $88 \%$ de los pacientes de este estudio evidenció coexistencia de dos o más síntomas y en el $63 \%$ se objetivó tres o más síntomas, lo que concuerda con lo descrito por Wine et al., (2013), que consignaron porcentajes semejantes $(88 \%=2$ o más síntomas y $69 \%=30$ más síntomas) $)^{(4-6)}$.

Gardella et al., también encontraron comorbilidades asociadas en la patogénesis de las alteraciones respiratorias en 12 de los 28 pacientes (43\%): Enfermedad por reflujo gastroesofágico, sensibilización atópica,
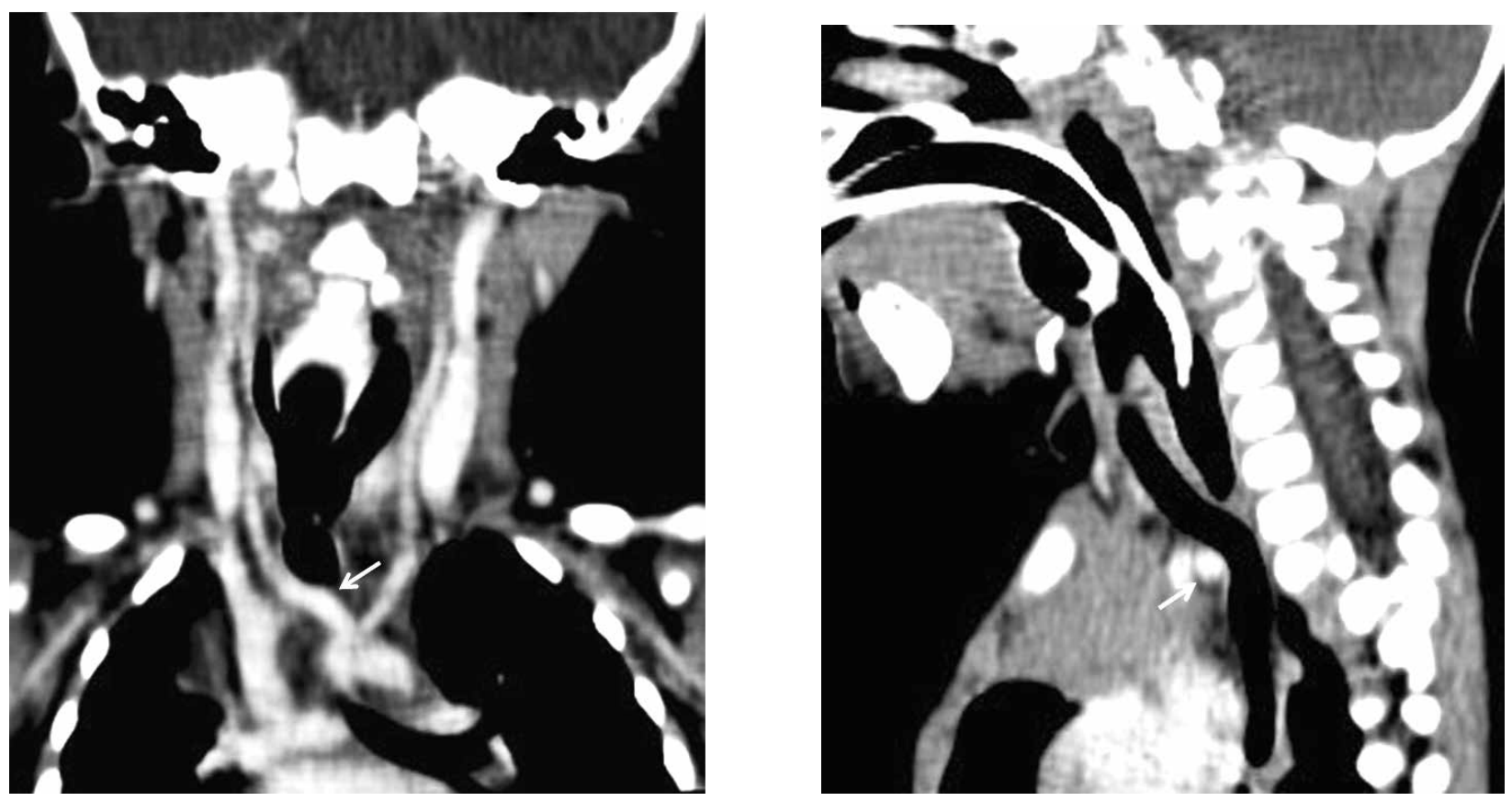

Figura 4. Imágenes de Caso $N^{\circ}$ 2. Compresión e incurvación traqueal por arteria innominada en TC cervical, con contraste (flechas); Reconstrucciones coronal (a) y sagital (b). 
asma, hiperreactividad bronquial, laringomalacia y paroxismo emocional ${ }^{(4)}$.

El diagnóstico de compresión traqueal por arteria innominada puede ser difícil de establecer clínicamente en pacientes sintomáticos, por las comorbilidades asociadas que estos presentan. Se describe diagnóstico erróneo inicial hasta en un $60 \%$ de los pacientes e importante retraso en establecer el diagnóstico correcto, de meses 0 años ${ }^{(4)}$, por lo que se ha establecido como imperativo efectuar un estudio de imagen planar, TC o resonancia magnética $(\mathrm{RM})^{(11)}$, además de fibrobroncoscopía en todo paciente con clínica sugerente de compresión traqueal ${ }^{(4)}$. La TC multicorte helicoidal con contraste endovenoso permite efectuar reconstrucciones que muestran con claridad la relación anatómica de la tráquea con la arteria innominada y además posibilita la adquisición de un estudio dinámico que objetive el colapso de la vía aérea.

En niños con estridor, la radiografía de tórax debe incluir proyecciones frontal y lateral; se describe que la indentación traqueal anterior puede ser visible hasta en un $30 \%$ de los niños con síndrome de compresión traqueal por arteria innominada ${ }^{(5)}$. Es importante destacar que el esofagograma no tiene indicación en esta patología porque, a diferencia de otras compresiones vasculares, resulta siempre normal debido a que no hay relación anatómica del esófago con la arteria innominada ${ }^{(3)}$. La fibrobroncoscopía permite una evaluación completa de la vía aérea, posibilitando la observación directa de la compresión traqueal u otra anomalía existente y definiendo su severidad, lo que ayuda a decidir la necesidad de tratamiento ${ }^{(4,9)}$.

El tratamiento de la compresión traqueal por arteria innominada puede ser conservador o quirúrgico. El tratamiento médico conservador habitualmente se realiza en aquellos pacientes en que la fibrobroncoscopía evidencia compresión no superior al $70 \%$ del diámetro de la tráquea y se basa en el manejo de las comorbilidades. El tratamiento quirúrgico se indica cuando los síntomas son severos o múltiples, no responden a tratamiento conservador y cuando la fibrobroncoscopía muestra compresión traqueal mayor al 70\%; el procedimiento quirúrgico de elección es la aortopexia ${ }^{(4)}$, que consiste en la sutura de la adventicia de la aorta al esternón, efectuada con evaluación simultánea del lumen traqueal mediante fibrobroncoscopía ${ }^{(6)}$.

Los resultados quirúrgicos son satisfactorios; Wine et al., reportaron que todos los pacientes presentaron una mejoría subjetiva con la aortopexia, con tasa de respuesta completa de $62 \%$ y tasa de respuesta parcial de $38 \%$ lo que está de acuerdo con los resultados de otros estudios que reportan tasas de respuesta completa entre $57 \%$ y $100 \%{ }^{\left({ }^{8}\right)}$. La frecuencia de complicaciones de esta cirugía es baja y varía entre 0 y $12.5 \%$, entre las que se consignan: derrame pleural o pericárdico, atelectasias, hemorragias y parálisis del nervio frénico ${ }^{(6,7)}$.

\section{Conclusiones}

La compresión traqueal por arteria innominada se observa mayoritariamente en sujetos asintomáticos. Cuando se presenta clínicamente como un síndrome, con síntomas respiratorios y/o comorbilidades, las imágenes cumplen un importante rol diagnóstico por lo que los radiólogos deben reconocer la alteración y evitar la ejecución de exámenes innecesarios en estos pacientes. La fibrobroncoscopía juega un rol fundamental en la evaluación directa de la patología y la alteración mecánica secundaria.

\section{Bibliografía}

1. Powell AJ, Mandell VS. Vascular rings and slings. En: Nadas' Pediatric Cardiology. Philadelphia: Pa. SaundersElsevier. 2nd ed. 2006. p. 811-22.

2. Yedururi S, Guillerman R, Chung T, Braverman R, Dishop M, Giannoni C et al. Multimodality Imaging of Tracheobronchial Disorders in Children. Radiographics 2008 May-Jun; 28(3): e29.

3. Siegel M, Smithuis R. Vascular Anomalies of Aorta, Pulmonary and Systemic vessels. http://www.radiologyassistant.nl/en/p4718c7f2eb7cc/vascular-anomalies-ofaorta-pulmonary-and-systemic-vessels.html. 30-07-2014.

4. Valletta E, Pregarz M, Bergamo-Andreis I, Boner A, Tracheoesophageal Compression Due to Congenital Vascular Anomalies (Vascular Rings). Pediatric Pulmonology 1997; 24: 93-105.

5. Berdon W. Rings, Slings, and Other Things: Vascular Compression of the Infant Trachea Updated from the Midcentury to the Millennium - The Legacy of Robert E. Gross, MD, and Edward B. D. Neuhauser, MD. Radiology 2000; 216: 624-632.

6. Gardella C, Donata G, Rossi G, Silvestri M, Tomá P, Bava $\mathrm{G}$ et al. Tracheal compression by aberrant innominate artery: clinical presentations in infants and children, indications for surgical correction by aortopexy, and short- and long-term outcome. Journal of Pediatric Surgery 2010; 45: 564-573.

7. Strife J, Baumel A, Dunbar J. Tracheal compression by the innominate artery in infancy and childhood. Radiology 1981; 139: 73-75.

8. Wine T, Colman K, Mehta D, Maguire R, Morell V, Simons J. Aortopexy for Innominate Artery Tracheal Compression in Children. Head and Neck Surgery July 2013; 149: 151-155.

9. Torre M, Carlucci M, Speggiorin S, Elliott M. Aortopexy for the treatment of tracheomalacia in children: review of the literature. Italian Journal of Pediatrics 2012; 38: 62.

10. Fawcet S, Gomez A, Hughes J, Set P. Anatomical variation in the position of the brachiocephalic (innomate artery) with respect to the trache: A computed tomography based study and literature review of innominate artery compression syndrome. Clinical Anatomy 2010; 23: 61-69.

11. Hellinger J, Daubert M, Lee E, Epelman M. Congenital thoracic vascular anomalies: Evaluation with State-ofthe-Art MR imaging and MDCT. Radiol Clin N Am 2011 49: 969-996. 